\title{
Erinnern nach der Erinnerung
}

Der Erste Weltkrieg in der Gegenwartsliteratur

\section{Erleben}

Bevor ich zu meinem eigentlichen Thema komme, dem Erinnern nach der Erinnerung in Gegenwartstexten zum Ersten Weltkrieg, werde ich als Kontrastfolie kurz beschreiben, worauf meines Erachtens Texte, die direkt nach dem Ersten Weltkrieg erschienen, reagierten. Was waren ihre Motive? Aus welcher Position sind sie geschrieben? Wie legitimieren sie sich als Fiktion über ein historisches Ereignis? Welche poetologische Funktion besitzt die Zeitzeugenschaft?

Der moderne Krieg ist ein radikaler Bruch mit allem, was vorher galt. Er zerstört sicher geglaubte Ordnungen und setzt, so Oliver Lubrich, Individuen und Gesellschaften der »Zumutung " aus, sich ein Ereignis, das »allen konventionellen Versuchen der Sinngebung " widerspricht, »begreiflich [zu] machen und das vollkommen Irrsinnige in den Kontext ihrer Erfahrung [zu] integrieren $\ll^{1}$

Insbesondere für die Soldaten, die die Schriftsteller der sersten Generation` häufig waren, brechen, so Bernd Hüppauf, extreme Gewalt,
Fiktionales Erinnern ohne zeitzeugenschaftliche Instanz, die eine tatsächliche Erinnerung an den Ersten Weltkrieg hätte, lässt sich auf die Formel , Erinnern nach der Erinnerung، bringen. Der Beitrag untersucht die narrativen Strukturen und erörtert die poetologischen Konsequenzen des ,Erinnerns nach der Erinnerung، in Erwin Mortiers Götterschlaf (2010), Jean Echenoz' 14 (2012) und Hans von Trothas Czernin oder wie ich lernte, den Ersten Weltkrieg zu verstehen (2013). 
Schrecken und Sinnlosigkeit irritierenderweise nicht nur von außen auf sie herein, sondern sie erfahren sich selbst an der Front auch als Auslöser dieser Situation. Die Konstellation, passiv Schrecken erdulden zu müssen und aktiv für Schrecken verantwortlich zu sein, verschärft das Erinnerungsund Artikulationsproblem. Zu den Versuchen von Soldaten, ihre ambivalenten Erlebnisse im Krieg in Briefen oder Tagebucheinträgen sinnhaft zu machen, bemerkt Joana Bourke, dass es dabei weniger darum gehe, das Kriegserlebnis diskursiv einzuholen, als darum, »eine Sprache zu finden nicht um der `Realität gleichzukommen`, sondern um jene Realität in den Ablauf des eigenen Lebens einzugliedern. Der Akt des Erzählens mag es dem Täter erlauben, seine kriegerischen Handlungen in sein Ich der Friedenszeit zu integrieren.$^{2}$ Diese Ausgangslage - Sinnverlust und Sinnsuche - führt sowohl zur Schreibwut als auch zur Sprachkrise. Schreiben und Nicht-Schreiben-Können konturieren sich gegenseitig. Es entsteht schon während des Krieges und dann vor allem nach 1918 eine regelrechte Flut an Kriegstexten, die vor der Aufgabe stehen, eine nicht einholbare, traumatische Realität als eben nicht artikulierbaren Schrecken versprachlichen und damit artikulieren zu wollen.

Auch in den Gegenwartstexten, auf die ich später genauer eingehen werde, wird dieser typische Topos - die Sprachkrise angesichts traumatischer Erfahrungen, Kommunikation des Inkommunikablen - aufgegriffen, so heißt es bspw. in Götterschlaf:

Als ich in den Jahren nach dem Krieg zum ersten Mal Proust las, verursachte es mir geradezu Bauchweh. Nicht die Zeit, die große Zeit hörte ich durch seine Sätze rauschen [...] Ich hörte die Sirenen der Sanitätswagen, die Räder der Krankenhausbetten über unebene Bodenfliesen rumpeln, die hastigen Schritte der Sanitäter und klimpernde Skalpelle und Wundklammern [...], die Schreie und das langgezogene Wimmern im größten Feldlazarett der Literatur [...]. Ich konnte lange kein vernünftiges Wort zu Papier bringen. ${ }^{3}$

Anders allerdings als beispielsweise bei Céline, bei dem der Erzähler, seine Erzählung und seine Sprache und damit `histoire und `discours ‘ irreversibel vom Trauma des Krieges infiziert bleiben werden und, wie Hans Ulrich Gumbrecht zeigt, die Gewalt in/von Célines Sprache auch auf den Leser geradezu körperlich übergreift, ${ }^{4}$ ist die Verunsicherung bei Helena, Mortiers Protagonistin, erstens zeitlich begrenzt und lässt zweitens die Form des Textes unangetastet. Die Schreibkrise ist für die Protagonistin überwindbar, die Wut der Erzählerin greift nicht auf den Text über und verbleibt so

4 Vgl. Gumbrecht: Louis-Ferdinand Céline. 
für den Leser auf inhaltlicher Ebene. Die schrecklichen Ereignisse auf der 'histoire<-Ebene bleiben diegetisch eingehegt, sie schlagen sich nicht auf die Sprache und nicht auf die >discours--Ebene durch:

Ich konnte lange kein vernünftiges Wort zu Papier bringen, wütend, wie ich war, ein großes schmollendes Kind, das die Lippen zusammenpresst und die Welt mit rot angelaufenem Gesicht vorwurfsvoll angafft, in der Hoffnung, genauso idiotisch zu wirken wie sie. Bis ich einsah, dass Schreiben die einzige Möglichkeit ist, dem Schweigen der Welt das eigene entgegenzusetzen. ${ }^{5}$

Es scheint, als ob die fehlende Zeitzeugenschaft auch eine sprachliche Dis$\operatorname{tanz}$ zu der erzählten Welt ermöglicht. Ohne Zeitzeugenschaft lassen sich 'histoire « und `discours « sauber trennen und die Sprache kann ihr Erzähltes kontrollieren, sie lässt sich nicht von den Schrecknissen affizieren. ${ }^{6}$

Aber auch Mortiers Protagonistin kann die Schönheit des Krieges aus sicherer Distanz durch den Blick durchs Teleskop genießen:

In jenem ersten Jahr war der Krieg in meinen Augen etwas Herrliches, der Pulsschlag eines einzigartigen Geschehens, der in allen Dingen klopfte. Ich spürte den Donner der Geschützrohre wie Druckwellen in meinem Zwerchfell und sah die Staubwolken über den Feldern, wenn ich in unserem Sommerhaus in Frankreich durch die Dachfenster nach dem Land von Artois Ausschau hielt [...].

Anders als in Texten von Zeitzeugen, bei denen Versuche, eine klare Außenposition einzunehmen, immer wieder durch traumatisches Einbrechen der Kriegsgewalt gestört werden, bleibt Mortier durchgehend auf Distanz. ${ }^{8}$ Das technische Medium dient hier schlicht als Apparat, dass nicht nur auf Distanz hält, sondern das Gesehene/Geschehene auch verharmlost. Mithilfe des Teleskops werden Wahrnehmung und Bewusstsein so kontrolliert bzw. in die Lage versetzt, selbst die Welt kontrollieren zu können, dass der Beobachtungsgegenstand (der Krieg) völlig problemlos in eine Sommeridylle integriert werden kann. ${ }^{9}$

5 Mortier: Götterschlaf, S. 33.

6 In Hans von Trothas Czernin oder wie ich lernte, den Ersten Weltkrieg zu verstehen verweist bereits der Titel auf den `Mehrwert` der Nichtzeitzeugenschaft. Zwar stört die Geschichte des Urgroßvaters immer wieder das Leben seines Urenkels Max, der sich gezwungen sieht, diese Geschichte zu recherchieren und sich mit den Erinnerungen des Grafen Czernin auseinanderzusetzen, das Resultat dieser Störung ist allerdings kein Kontrollverlust, sondern, im Gegenteil, , Verstehen`.

7 Mortier: Götterschlaf, S. 112.

$8 \mathrm{Zu}$ Versuchen der ästhetischen Distanzierung bei Jünger vgl. Kirschstein: Writing War, insb. S. 96-111.

9 Wie anders ergeht es da E.T.A. Hoffmanns Nathanael, der nach dem Blick durch das Perspektiv weder sich noch seine Objekte unverzerrt wahrnehmen kann. Aus Puppen werden lebendige Frauen und aus lebendigen Frauen Puppen. Aus einem Studenten und Dichter wird ein wahnsinniger Selbstmörder (siehe Hoffmann: Der Sandmann). 
Ausgehend von der eingangs beschriebenen Situation - Krieg als Zivilisationsbruch - lassen sich die Texte der >ersten Generation` als Reaktion auf eine radikale Situation der nicht mehr in bekannte Deutungsmuster zu bringenden Erfahrung des modernen kriegerischen Gewaltszenarios und zugleich als Konstruktion solch einer Situation beschreiben. ${ }^{10}$ Zentral sind dabei Darstellungen von Regressionserfahrungen, radikales NichtVerstehen und nichtkontrollierbare Affekte des eigenen Körpers. Gleichzeitig lassen sich Kriegstexte auch als eine Art Reinigungsprozedur lesen, in der die destabilisierenden Erfahrungen des Krieges einerseits gebannt, andererseits entfesselt, beschworen und auch verehrt werden.

Das alles findet nun in der Kriegsliteratur der sersten Generation ‘ von einer (Erzähl)Position aus statt, die man als steilnehmende Beobachtung beschreiben kann. Die Autoren kämpften vielfach im Ersten Weltkrieg und legitimierten ihr Schreiben und ihr Wissen über diese Teilnahme und das unmittelbare Erleben des Krieges, das es weiterzugeben galt. Sie sind nicht nur Zeitzeugen, ihre Zeugenschaft ist teilnehmend involviert.

Diese teilnehmende Beobachtung ist eng mit Erleben und Ereignishaftigkeit korreliert. Es entsteht also die Idee, dass derjenige, der im Krieg stand, der involviert und teilnehmend beteiligt war, den Krieg gerade deshalb besonders authentisch wiedergeben kann. Leschke fasst diese Konzentration auf den Krieg als Ereignis sehr negativ, indem er konstatiert:

Kriegserzählungen signalisieren [...] eine besondere Nähe zu den erzählten Ereignissen und sie legen dabei ein ebenso urwüchsiges wie naives Widerspiegelungsmodell zu Grunde: Der Narration selbst wird, sofern sie nur hinreichend kunstlos betrieben wird, kein großer Einfluss zugetraut, das Entscheidende sind vielmehr die Ereignisse, auf denen die Erzählung basiert. ${ }^{11}$

Auch wenn ich Leschkes generalisierender Kritik nicht folge, ist die Beobachtung, dass sich Kriegstexte der sersten Generation` ganz zentral über die Nähe zu den erzählten Ereignissen definieren und legitimieren, offensichtlich korrekt. Das lässt sich sowohl für die Texte selbst als auch für die Rezeption sagen. Das auf der Ebene der Rezeption bis heute immer wiederkehrende Anliegen einer Abgleichung von Darstellung und ‘realem Krieg، als scheinbar außerhalb des Textes liegendem Referenten, beziehungsweise von Autorbiographie und Handlung, korrespondiert vielfach auf der Ebene des Textes mit den verschiedensten Authentizitätsstrategien und -effekten. So heißt es beispielsweise in Jüngers Kampf als inneres Erlebnis:

10 Vgl. dazu Lubrich: Das Schwinden der Differenz und Kirschstein: Writing War.

11 Leschke: Kriegerische Opfer, S. 99. 
Was soll ich Eure Nerven schonen? Lagen wir nicht selbst einmal vier Tage in einem Hohlweg zwischen Leichen? Waren wir nicht alle, Tote und Lebendige, mit einem dichten Teppich großer, blauschwarzer Fliegen bedeckt? Gibt es noch eine Steigerung? Ja: es lag dort mancher, mit dem wir manche Nachtwache, manche Flasche Wein und manches Stück Brot geteilt hatten. Wer darf vom Kriege reden, der nicht in unserm Ringe stand? ${ }^{12}$

Jüngers Text positioniert sich hier, ganz im Sinne von Jan Assmanns Beschreibung des kulturellen Gedächtnisses, selbst als Medium kollektiver Identitätsbildung einer bestimmten Gruppe, der Frontsoldaten, denen qua körperlicher Anwesenheit und physischem und psychischem Leiden die Erinnerungshoheit am zu erinnernden Ereignis zugesprochen wird. ${ }^{13}$

Literarische Texte können dabei, das hat Pierre Nora in seinen Lieux de memoire zumindest angedeutet, zu Erinnerungsorten werden, die zum Zwecke der Deutung von Vergangenem und Gegenwärtigem aktiv angeeignet bzw. generiert werden können. ${ }^{14}$ Dabei können bestehende nationale Selbstbilder oder kollektive Werte affirmiert oder auch einer kritischen Reflexion unterzogen und durch Gegenerinnerungen kontrastiert werden. Jüngers Text wird in diesem Sinne zum Erinnerungsort, der die Identität und den Fortbestand der Gruppe über den Tod ihrer Mitglieder hinaus sichern und gegen Fehlinterpretationen von außen immunisieren soll. Der Leser wird dabei in die Position eines Beobachters oder fast Voyeurs verschoben, dem ein Einblick in ein Ereignis gewährt wird, von dessen Deutung er ausgeschlossen bleibt.

Die hier deutlich werdende Ebene der Erfahrung und des Erlebens lässt sich für Kriegsliteratur der ersten Generation als zentral für die Möglichkeiten ihrer Repräsentation konstatieren. Wie `naiv`, wie Leschke kritisiert, oder `selbstreflexiv`, wie ich das an vielen Texten beobachten würde, dann mit diesen Möglichkeiten umgegangen wird, ist eine Frage, die in diesem Rahmen zu weit führen würde. Bestenfalls nutzen die Texte diese Nähe als Literatur und werden, so Neumann und Weigel, "zum Organon von Differenzierung, Übersetzung und Verschiebung, von Fortschreibung, Transgression von Grenzen und Bruch mit der symbolischen Ordnung und den codierten Bedeutungen $«{ }^{15}$ Oder, um das in Richtung unseres Themas zu übersetzen, sie versuchen gerade nicht, den Krieg widerzuspiegeln oder

12 Jünger: Der Kampf als inneres Erlebnis, S. 22.

13 Vgl. Assmann: Das kulturelle Gedächtnis, S. 39: »ede Gruppe, die sich als solche konsolidieren will, ist bestrebt, sich Orte zu schaffen und zu sichern, die nicht nur Schauplätze ihrer Interaktionsformen abgeben, sondern Symbole ihrer Identität und Anhaltspunkte ihrer Erinnerung. Das Gedächtnis braucht Orte, tendiert zur Verräumlichung." 
auszudeuten, sondern führen das Scheitern angesichts jeder - nicht nur literarisch - erzählten Wirklichkeit des Krieges vor.

\section{Erinnern}

Ich komme nun vor diesem Hintergrund zu meinem eigentlichen Thema, der gegenwärtigen Literatur zum Ersten Weltkrieg, und beginne mit einigen Überlegungen zur Funktion von Gedächtnis und Erinnerung. Gedächtnis und Erinnern spielen, das ist inzwischen ein Gemeinplatz der Gedächtnisforschung, eine entscheidende Rolle bei Aufbau und Erhalt individueller wie gesellschaftlicher Identität. Dabei hängen Gedächtnis und Erinnerung nicht von Vergangenheit ab, sondern Vergangenheit gewinnt erst durch die Modalitäten des Erinnerns Konturen. Denn, wie Nicolas Pethes sehr schön formuliert: »Das Problem des Gedächtnisses ist [...] seine Leere. Das, worum es ihm geht, das Vergangene, ist qua Vergangensein nicht verfügbar. « ${ }^{16}$ Die Aufgabe der Erinnerung ist nun, diese nicht erreichbare Vergangenheit für individuelle, kollektive oder kulturelle Gedächtnisse zu konstruieren. Das betrifft selbstverständlich nicht nur individuelles Erinnern, sondern auch historiographisches Erinnern, das nicht etwa `die< Vergangenheit darstellt, sondern eine Vergangenheit durch Rekurs auf Zeugnisse in erzählenden Sinnzusammenhängen herstellt.

Erinnern als aktuelle Sinnproduktion wird, das wissen wir spätestens seit Hayden Whites Metahistory, stark von gestaltendem Erzählen beeinflusst. Beide (Erinnern und Erzählen) scheinen denselben Mustern kohärenter Konstruktion von Zusammenhängen zu folgen. Beide stellen einen (auch fiktiven) Zusammenhang zwischen einem Ereignis und seiner Repräsentation in der erzählten Erinnerung her. Dabei folgen die Erzählschemata nicht einer intrinsischen Wahrheit des Ereignisses, sondern den Kohärenz- und Konsistenzerwartungen einer Erzählgemeinschaft oder Gesellschaft. »Im Erinnern«, so Siegfried J. Schmidt, »wird Erlebtes so mit Erzähltem verbunden, dass endgültig verloren geht, was man als `wirkliche Quelle oder `reales Erlebnis bezeichnen könnte. $\ll^{17}$ Gerade dieser fehlende Ursprung dessen, was erinnert wird, macht nun literarische Texte, so Wilhelm Voßkamp, als »Medien des kommunikativen wie kulturellen Gedächtnisses, als eine Art Reflexionsinstanz, als ein semantisches und vor allem performatives Archiv« 
attraktiv. ${ }^{18}$ Literatur kann, wie Carsten Gansel und Pawel Zimniak es tun, als Medium verstanden werden, mit dessen Hilfe »in Form von narrativen Inszenierungen individuelle und generationenspezifische Erinnerungen für das kollektive Gedächtnis bereitgestellt werden. $«^{19}$ Mehr noch, über die Art und Weise der narrativen Inszenierung in literarischen Texten kann auf die Art und Weise der in einer Gesellschaft ablaufenden Prozesse der Gedächtnisbildung geschlossen werden.

Gleichzeitig erschöpft sich das kulturelle Gedächtnis nicht in der Sicherung zu erinnernder Inhalte, sondern Gedächtnismedien wie bspw. Räume, aber auch Texte, können dazu beitragen, ein neues oder anderes Gedächtnis zu konstruieren:

Die Funktion des kulturellen Gedächtnisses ist mitnichten auf Bewahren, Kontinuität, Identität und Erinnern gegründet. [...] Tatsächlich ist die Operation des Gedächtnisses die Unterscheidung von Erinnern und Vergessen, die Selektion von aktuell Erinnertem aus potentiell Erinnerbarem. Das Gedächtnis ist nichts anderes als diese Unterscheidung, dieser Grenze. [...] Die Potentialität des aktuell nicht Erinnerten bleibt dem kulturellen Gedächtnis als Potential erhalten. Entsprechend ist Kultur selbst nichts als die Instanz der Auswahl, der Prozess des stets neuen Ziehens der Grenze zwischen Erinnern und Vergessen $\left[\ldots . . . .^{20}\right.$

Genauso fungiert auch Literatur als Gedächtnismedium nicht einfach als Mittel zur Konstruktion von mehr oder weniger haltbarer Erinnerung, sondern als Instanz, die diese Grenze zwischen Erinnern und Vergessen auslotet, immer wieder neu justiert und vor allem diese Grenze selbst, die Art und Weise unseres Erinnerns und Vergessens sowie die Unterscheidung zwischen aktuell und potentiell Erinnertem, sichtbar macht.

\section{Erzählen}

Was ich vor diesem Hintergrund im Folgenden exemplarisch veranschaulichen möchte, ist, dass das Thema der aktuellen Texte zum Ersten Weltkrieg (auch wenn ihnen vom Feuilleton, wie hier in einem Beitrag zu Götterschlaf, attestiert wird, sie führten uns "tief in den Alltag des Ersten Weltkrieges hinein, und dies so eindrucksvoll, als habe der Autor jene Zeit selbst erlebt $^{21}$ ) weder das Erlebnis noch die Folgen dieses Krieges sind, sondern

19 Gansel/Zimniak: Das »Prinzip Erinnerung", S. 13.

20 Pethes: Diesseits der Leitha, jenseits der Lethe, S. 3. Zur Funktion des Vergessen für Erinnerung und Gedächtnis s. Esposito: Soziales Vergessen.

21 Brandt: In den Schützengräben verrät sich der Mensch. 
dass sie vielmehr den Vorgang des Erinnerns und Vergessens selbst ins Zentrum rücken. Die Texte fragen nicht: Was wird erinnert?, sondern: Wie wird erinnert? bzw. Was sind die Bedingungsmöglichkeiten des Erinnerns, nachdem die Zeugen und teilnehmenden Beobachter des Ersten Weltkriegs nicht mehr zur Verfügung stehen?

Arbeiten sich also die Texte der ersten Generation daran ab, im/am Erlebnis des Krieges Sinn zu generieren oder wiederzugewinnen, den Krieg darstellbar zu machen, oder seine Undarstellbarkeit vorzuführen, geht es den gegenwärtigen Texten darum, auszuloten, wie der Krieg im kollektiven und kulturellen Gedächtnis erinnert wird bzw. erinnert werden kann oder auch sollte. Ich gehe davon aus, dass die zeitgenössischen Zeitzeugentexte und die gegenwärtigen Kriegstexte ihre Funktion als Erinnerungsmedium jeweils unterschiedlich einlösen. Mit Zeitzeugenschaft ist mitnichten gemeint, dass die Erinnerungsarbeit authentisch wäre. Auch Texte der ersten Generation sind fiktionale Konstruktionen und ihre Authentizität genauso abhängig von ihrer Literarizität und Poetizität wie von der zugrundeliegenden erlebten Kriegserfahrung. Der Unterschied zwischen den Texten der ersten Generation und aktuellen Texten zum Ersten Weltkrieg ist nicht allein an der Zeitzeugenschaft und teilnehmenden Beobachtung abzumessen, sondern an den poetisch unterschiedlichen Verfahren, mit denen im ersten Fall Zeitzeugenschaft literarisch verarbeitet und im zweiten Fall literarische Erinnerungsarbeit ohne Zeitzeugenschaft geleistet wird.

Für Gegenwartsliteratur generell wird eine wichtige Zäsur um 1989 gesetzt - mit den globalen Veränderungen/Verschiebungen ist es zu einem

Umbau des >Funktionsgedächtnisses insofern gekommen, als eine Neuaufnahme und Neubewertung erfolgt. Es gelangen nunmehr auch jene Vorgänge, Themen, Spuren ins lebendige Gedächtnis, die über einen längeren historischen Zeitraum ausgeblendet, abgewiesen, ausgemustert oder verworfen waren. Krieg und Holocaust erscheinen ebenso in einem anderen $>$ Licht $`$ wie Flucht, Vertreibung oder Bombenkrieg. ${ }^{22}$

Es geht in der Folge dieser Zäsur aber eben nicht nur darum, was erinnert wird bzw. jetzt erinnert werden kann, sondern das Erinnern selbst gerät in den Fokus - Gansel spricht vom »Reflexivwerden der Erinnerung « ${ }^{23}$ Etablierte Modi der Erinnerung müssen unter veränderten historischen Bedingungen neu bedacht oder dekonstruiert werden. Wir befinden uns hier nicht auf der Ebene der Epistemologie des Erinnerten, sondern auf der Ebene der Epistemologie des Erinnerns. 
Dass die Zäsur 1989 nicht nur im Hinblick auf den Zweiten Weltkrieg, sondern auch auf den Ersten relevant war, kann man nicht zuletzt in der Geschichtswissenschaft prominent an Christopher Clarks The Sleepwalkers ablesen, der das, was er »The Balkan inception scenario $\aleph^{24}$ nennt, von der Peripherie ins Zentrum rückt und dabei erstens versucht, das bislang tendenziell vorherrschende `James-Bond-Muster in ein gesamteuropäisches Bild umzuschreiben und zweitens die Kontingenz der Handlungen und Handlungsketten deutlich macht, die in die Katastrophe geführt hatten. Auch hier finden wir also ein Umschalten von der Frage, warum der Krieg ausbrach, auf die Frage, wie es dazu kam.

Ein erstes deutliches Indiz für diese Verschiebung vom Krieg auf seine Erinnerung ist das Fehlen eines in den Texten der ersten Generation so auffälligen Bruches. Jünger inszeniert die erste Begegnung mit dem Krieg als radikale Differenzerfahrung - in In Stahlgewittern lesen wir: »Mit Entsetzen ahnst du, daß deine ganze Intelligenz, deine Fähigkeiten, deine geistigen und körperlichen Vorzüge zur unbedeutenden, lächerlichen Sache geworden sind. $\ll^{25}$ Mortiers Helena dagegen bekennt lange nach Kriegsbeginn schuldbewusst: »eigentlich ist der Krieg das Beste, das mir widerfahren ist «, und ihr Bruder, gerade von der Front zurückgekehrt, stimmt ihr zu: »Weißt du was, meine kleine Gazelle? [...] mir auch. ${ }^{26}$ Was wir hier lesen, ist nicht als Darstellung naiver Kriegsbegeisterung oder Blindheit gegenüber den Schrecken des Krieges zu verstehen. Solche bekommen wir durchaus auch präsentiert: durchs Teleskop oder über die Betrachtung alter Kriegsfotografien perspektivisch gebrochen. ${ }^{27}$ Was hier auf der Handlungsebene vielmehr retrospektiv für die Entwicklung der Protagonisten positiv gedeutet wird - Helena befreit der Krieg von den sie einengenden Familienstrukturen, ihrem Bruder gibt er die Gelegenheit, seine Homosexualität zu leben -, macht auf der Ebene der Erzählung eine Verschiebung vom Schockerlebnis des Krieges hin zu der Spur deutlich, die er in die Erinnerung seiner Protagonisten zieht:

die Sehnsucht ist da [...], den Stift zu einer Pflugschar umzuschmieden und ein gerade beschriebenes Blatt wieder umzupflügen, quer zu den Zeilen, Furche nach Furche. Dann würde ich auf einen blanken Acker zurückblicken, auf all das, was der Pflug zutage gefördert

27 Vgl.: »Drei andere Gestalten [auf dem Bild] lösen sich unerkennbar in dem dichten Nebel auf, der durch die Baumstämme über den Grabenrand in den Unterstand zu sichern scheint - ein milchiger Dunst, der mich immer wieder mit einem leichten Grausen erfüllt, weil er mich an Giftgas erinnert.« (ebd., S. 275) 
hat: durchgerostete Eimer, Stacheldraht, Knochensplitter, Bettstäbe, einen Blindgänger, einen Ehering. Ich würde viel darum geben, in die Tiefen unserer Geschichten absteigen zu können, an einem Seil in ihre dunklen Schächte herabgelassen zu werden und Erdschicht um Erdschicht im Lampenlicht vorbeigleiten zu sehen. Alles, was der Boden in sich aufgenommen hat. Fundamente, Zaunpfähle, Baumwurzeln, Suppenteller, Soldatenhelme, die Skelette von Tieren und Menschen in einem zum Stillstand gekommenen Chaos, dem zu Erdkruste erstarrten Mahlstrom, der uns verschlang. ${ }^{28}$

Der Text wird hier im Text als Arbeit an der Erinnerung beschrieben, der die teilweise traumatischen Gedächtnisfragmente des Krieges ordnet, umgruppiert, wieder in Bewegung bringt. Im Fall von Helena ist das >Umpflügen der Erinnerung`gelungen - ihr Tod markiert das Ende der Erzählung, sie kann versöhnt mit ihrem Leben, ihrer Erzählung sterben - »es gibt nun nichts mehr zu erzählen $«{ }^{29}$ heißt es auf der letzten Seite des Textes. Dabei korreliert zum einen das Gelingen der Kriegsbewältigung mit der Metapher des Pflügens. Der gepflügte Acker verheißt in der Herausarbeitung des Alten (»Fundamente, Zaunpfähle, Baumwurzeln, Suppenteller, Soldatenhelme, die Skelette von Tieren und Menschen «) für die Zukunft eine neue Ernte, die zwar nicht Helena, aber ihre Leser einfahren können. Der gepflügte Acker ist trotz oder aufgrund des Hervorhebens des Alten und Abgestorbenen ein Fruchtbarkeitsbild. Die Erinnerungsarbeit ist zum anderen aufgrund der Metapher des Pflügens buchstäblich ergodisch. Erinnern wird als Ergon ausgewiesen, als Werk, das als Ergebnis des Ackerns (buchstäblich und metaphorisch) ausgewiesen wird.

Auch im Fall von Hans von Trothas Czernin wird deutlich, dass nicht mehr, wie in den zeitgenössischen Texten, seinfach ‘ an Erlebtes erinnert werden kann, sondern Erinnerung immer Arbeit an der Erinnerung bedeutet. Max, eigentlich Ottokar von Andersleben, Urenkel des historischen Grafen Ottokar Czernin, des letzten Außenministers der Doppelmonarchie, will mit seiner Familiengeschichte eigentlich nichts zu tun haben: »Für ihn war Max das Gegenteil von Ottokar. Die drei Silben klangen in seinen Ohren wie ein schweres Echo aus einer düsteren Zeit. Und Max wollte leben, jetzt. « ${ }^{30}$ Und doch führt ihn eine geheimnisvolle Begegnung in den Wirren des Mauerfalls 1989 zurück in die Geschichte einer weiteren historischen Zäsur - des Ausbruchs des Ersten Weltkriegs und zur Rolle seines Urgroßvaters dabei. Widerwillig nimmt Max den Auftrag seiner Großmutter an, die Geschichte ihres Vaters, seines Urgroßvaters, ins rechte Licht zu rücken: 
»Es geht doch um die Wahrheit. Darum, wie es eigentlich gewesen ist. «11 Anders als Mortiers Helena, die sich als Augenzeugin erinnert, wird hier schon durch die Figurenkonstellation - der Urenkel macht sich detektivisch auf die Suche nach der Geschichte seines Urgroßvaters - klar, dass es nicht um den Krieg als Ereignis, sondern um die Funktion der Erinnerung als Machtinstrument und um die Kontingenz historischer Wahrheit geht. Und so bleibt die Figur des Großvaters, genau wie dessen Geschichte, trotz aller akribischen Nachforschungen und Erinnerungskämpfe ein Geheimnis: »Irgendwann fragte Fiona >Was meinst du, wie er war? ? [...]. >Ich weiß es nicht. Es war die kürzeste Variante. $\aleph^{32}$

Ein weiteres Beispiel, um die verschiedenen Zielrichtungen der frühen Kriegstexte und der Gegenwartstexte veranschaulichen zu können, ist die gegensätzliche Verwendung der metaphorischen Verbindung von Musik und Front, die wir sowohl bei Jünger und Céline als auch bei Echenoz finden. In der Szene in In Stahlgewittern, die die Ankunft der Rekruten vor der Front beschreibt, heißt es: »mit ungläubiger Ehrfurcht lauschten wir dem langsamen Takte des Walzwerks der Front, einer Melodie, die uns in langen Jahren Gewohnheit werden sollte «. ${ }^{33}$ Die Szene lässt sich zu einer analogen Szene aus Voyage au Bout de la Nuit in Beziehung setzen. Auch für Bardamu steht Musik am Anfang des Krieges. Allerdings verstummt hier die Musik nach und nach, je mehr sich die Front nähert. Schließlich finden sich die Soldaten völlig alleine, ausgeliefert und orientierungslos im stummen Niemandsland des Krieges wieder. Während also in beiden Texten das Einsetzen bzw. Verstummen der Musik den Bruch zwischen ziviler Welt und Front markiert und ausdeutet, finden wir bei Echenoz durch eine von der Mobilmachung bis zum ersten Kontakt mit dem Feind absurderweise nie abbrechende Melodie einen fließenden Übergang zwischen den beiden Welten, der genau hierin die maßlose Gewalt des Krieges zeigt. Die Regimentskapelle, die den Angriff unterstützen soll, wird als eine Art automatischer Spieluhr gezeigt, aus der nach und nach immer mehr Teile herausfallen:

Es erschien eine zwanzigköpfige Gruppe von Männern und stellte sich, offenbar ohne jede Furcht vor Geschossen im Halbkreis auf. Das waren die Regimentsmusikanten, deren Dirigent die Marseillaise anstimmen ließ, indem er den erhobenen Stab senkte, in der Absicht, damit kühn den Angriff zu begleiten. [...] Während das Orchester seinen Teil zum Kampfgeschehen beitrug, erhielt der Arm des Baritonhorns einen Durchschuss, und die Posaune fiel, übel verwundet: Der Halbkreis rückte darüber zusammen, und die 
Musiker spielten, wenn auch in verminderter Formation, ohne jede falsche Note weiter, und als sie dann die Zeile wiederholten, in der das blutige Banner erhoben wird, fielen Flöte und Althorn tot zu Boden. ${ }^{34}$

Der Ton dieser Passage steht in seiner Verbindung von Lakonie und Drastik durchaus in celinscher Tradition, die Erzählpositionen unterscheiden sich aber deutlich. ${ }^{35}$ Bei Céline haben wir es mit einer zwar hochgradig problematischen und unzuverlässigen, aber doch strukturell angelegten Einheit von Autor, Erzähler und Protagonisten zu tun. Bei Echenoz hingegen werden wir immer wieder ostentativ auf die Nachträglichkeit des Erzählens gestoßen: So heißt es über einen Kameraden des Protagonisten, er »würde sich in der Zeit, die ihm noch zu leben blieb « ${ }^{36}$ nicht mehr an die zu große Uniformhose gewöhnen. Während also bei Céline der Schock durch die Gleichzeitigkeit, durch das Miterleben der traumatisierten Perspektive des Erzählers/Protagonisten auf den Leser überspringt, ist das Beunruhigende bei Echenoz die Erkenntnis, dass die distanzierte Perspektive keine Linderung, keine Entschärfung des Krieges bringt, sondern wir aushalten müssen, dass er auch hundert Jahre später in keine sinnstiftende Erzählung verwandelt werden kann. Auch das wird wieder in einem an celinschen Biologismus erinnerndem Bild einer Zeugung, die dem sanften Tod von Mortiers Protagonistin diametral entgegensteht, deutlich: »Er penetrierte und befruchtete sie. Und im Herbst danach, genau zur Zeit der Schlacht von Mons, die die letzte sein sollte, wurde ein Junge geboren, und man gab ihm den Vornamen Charles. « ${ }^{37}$

Literatur lässt sich bei Echenoz nicht im Sinne eines Erinnerungsmediums verstehen, das »Deutungsmuster zur Verfügung stellt, die die Funktion von Orientierungshilfen in einer komplexen Wirklichkeit übernehmen [...] und Kodierungsformate für nachfolgende Erfahrungen bereitstell[en] « ${ }^{38}$ kann. Im Bild des > Phantomschmerzes $\triangleleft,{ }^{39}$ das Echenoz sowohl für Anthimes verlorenen Arm als auch für seine Erinnerung ins Spiel bringt, wird sein

Echenoz: 14, S. 61.

Auffällig ist zudem die ironische Diskrepanz zwischen martialischer Marseillaise und stoischem Weiterspielen.

Echenoz: 14, S. 14.

Ebd.

Neumann: Erinnerung - Identität - Narration, S. 125.

Vgl.: »Die alte Sache mit dem Phantomglied. [...] Es kommt [...] vor, und das schien bei Anthime der Fall zu sein, dass die Präsenz einer solchen Gliedmaße lange nach deren Verlust in die Organisation des Körpers zurückkehrt.« (Echenoz: 14, S. 122) Auch in Götterschlaf heißt es: »Manchmal frage ich mich, ob all meine Erinnerungen diesen Namen noch verdienen, ob ihre Schärfe und Unmittelbarkeit sie nicht vielmehr zu Phantomschmerzen der Seele machen." (Mortier: Götterschlaf, S. 49) 
Text vielmehr zum Medium, das die Schrecken des Krieges nicht zu bannen oder in eine sinnhafte Erzählung einzuordnen versucht, sondern zeigt, wie der Verlust - von Körpern, Menschen, Erinnerungen - uns noch hundert Jahre später heimsuchen, an die Oberfläche drängen kann, ohne jemals ein- oder zurückgeholt werden zu können. Während Mortiers Helena mit dem gepflügten Acker auf Wachstum und Zukunft auf der Grundlage des Erinnerns setzt, zeigt Echenoz mit dem Phantomschmerz, wie Erinnern, und vielleicht gerade zeitzeugenschaftsloses Erinnern, Mangel und Abwesendes perpetuiert. Phantome verweisen nicht auf die Zukunft, sie sind immer eingefasst in ein Narrativ der Heimsuchung durch Vergangenes.

\section{Literaturverzeichnis}

Assmann, Jan: Nachwort. In: Elena Esposito: Soziales Vergessen. Formen und Medien des Gedächtnisses der Gesellschaft. Frankfurt/M.: Suhrkamp 2002, S. 400-414.

Assmann, Jan, Das kulturelle Gedächtnis. Schrift, Erinnerung und politische Identität in frühen Hochkulturen. München: C.H. Beck 1999.

Brandt, Sabine: In den Schützengräben verrät sich der Mensch. In: »FAZ«3.9.2010. <http:// www.faz.net/aktuell/feuilleton/buecher/rezensionen/belletristik/erwin-mortiergoetterschlaf-in-den-schuetzengraeben-verraet-sich-der-mensch-11014508.html> (Zugriff: 1.11.2015).

Bourke, Joanna: Auge in Auge mit dem Feind. Das Töten von Angesicht zu Angesicht in den Kriegen des 20. Jahrhunderts (1914-1975). In: Massenhaftes Töten. Kriege und Genozide im 20. Jahrhundert. Hgg. Peter Gleichmann, Thomas Kühne. Essen: Klartext 2004, S. 287-306.

Céline, Louis-Ferdinand: Reise ans Ende der Nacht [1932]. Reinbek: Rowohlt Verlag 2003.

Clark, Christopher: The Sleepwalkers. How Europe Went to War in 1914. London: HarperCollins 2012.

Echenoz, Jean: 14 [2012]. München: Carl Hanser Verlag 2014.

Gansel, Carsten; Zimniak, Pawel (Hgg.): Das »Prinzip Erinnerung « in der deutschsprachigen Gegenwartsliteratur nach 1989. Göttingen: Vandenhoeck \& Ruprecht 2010.

Gansel, Carsten: Formen der Erinnerung in der deutschsprachigen Gegenwartsliteratur nach 1989. In: Das »Prinzip Erinnerung « in der deutschsprachigen Gegenwartsliteratur nach 1989. Hgg. ders., Pawel Zimniak. Göttingen: Vandenhoeck \& Ruprecht 2010, S. 19-36.

Gumbrecht, Hans Ulrich: Louis-Ferdinand Céline und die Frage, ob Prosa gewaltsam sein kann. In: Kunst - Macht - Gewalt. Der ästhetische Ort der Aggressivität. Hg. Rolf Grimminger. München: Fink, S. 203-222.

Jünger, Ernst: In Stahlgewittern. Aus dem Tagebuch eines Stoßtruppführers. Hannover: Selbstverlag des Verfassers 1920.

Jünger, Ernst: Strahlungen II. Sämtliche Werke, Bd. III. Stuttgart: Klett-Cotta 1980.

Jünger, Ernst: Der Kampf als inneres Erlebnis. Sämtliche Werke, Bd. II,7. Stuttgart: KlettCotta 1980.

Kirschstein, Daniela: Writing War. Kriegsliteratur als Ethnographie bei Ernst Jünger, LouisFerdinand Céline und Curzio Malaparte. Würzburg: Königshausen \& Neumann 2014. 
Leschke, Rainer: Kriegerische Opfer: Von den Verlusten der Kriegserzählung. In: Imaginäre Welten im Widerstreit. Krieg und Geschichte der deutschsprachigen Literatur seit 1900. Hgg. Lars Koch, Marianne Vogel. Würzburg: Königshausen \& Neumann, S. 98-118. Lubrich, Oliver: Das Schwinden der Differenz. Postkoloniale Poetiken. Alexander von Humboldt - Bram Stoker - Ernst Jünger - Jean Genet. Bielefeld: transcript 2004.

Mortier, Erwin: Götterschlaf [2008]. Köln: DuMont 2010.

Neumann, Gerhard; Weigel, Sigrid: Einleitung. In: Lesbarkeit der Kultur. Literaturwissenschaft zwischen Kulturtechnik und Ethnographie. Hgg. dies. München: Fink 2000, S. 9-16.

Pierre Nora: Les Lieux de mémoire. Paris: Gallimard 1997.

Pethes, Nicolas: Diesseits der Leitha, jenseits der Lethe. Zehn Thesen zum Raumkonzept der kulturwissenschaftlichen Gedächtnisforschung. In: Leitha und Lethe. Symbolische Räume und Zeiten in der Kultur Österreich-Ungarns. Hgg. Amália Kerekes, Alexanda Millner, Peter Plener, Béla Rásky. Tübingen: Francke 2004, S. 1-18.

Schmidt, Siegfried J.: Gedächtnis und Gedächtnistheorien. In: Metzler Lexikon Literaturund Kulturtheorie. Dritte, aktualisierte und erweiterte Auflage. Hg. Ansgar Nünning. Stuttgart, Weimar: Metzler 2004, S. 216-218.

Trotha, Hans von: Czernin oder wie ich lernte, den Ersten Weltkrieg zu verstehen. Berlin: Nicolai 2013.

Voßkamp, Wilhelm: Die Gegenstände der Literaturwissenschaft und ihre Einbindung in die Kulturwissenschaften. »Jahrbuch der deutschen Schillergesellschaft« 42 (1998), S. 503-510.

Wimbauer, Tobias (Hg.): Ernst Jünger in Paris. Ernst Jünger, Sophie Ravoux, die Burgunderszene und eine Hinrichtung. Hagen-Berchum: Eisenhut Verlag 2011. 